\title{
Calculation of the Electric Field Based on Average Momentum Transfer Using Pixelated Electron Detector in STEM
}

\author{
Wenpei Gao ${ }^{1}$, Christopher Addiego ${ }^{2}$ and Xiaoqing Pan $^{1,2}$
}

${ }^{1 .}$ Department of Chemical Engineering and Materials Science, University of California, Irvine, ET 644, Irvine, CA, USA

2. Department of Physics and Astronomy, University of California, Irvine, 4129 Frederick Reines Hall, Irvine, CA, USA

Determination of the electric field at the atomic scale in materials will help better understand the properties of ferroelectric materials, light emitting diodes and heterogeneous catalyst. To map the electric field, differential phase contrast imaging (DPC) in STEM (scanning transmission electron microscopy) has been developed by using segmented electron detectors to collect scattered electrons. The variation in electron count among the detectors are related to the interaction between electrons and the built-in electric field in materials; when coupled with the high spatial resolution in aberration corrected STEM, DPC can reveal the electric field in $\mathrm{BaTiO}_{3}$ [1], GaAs nanowires and other nanostructures at atomic resolution. Recently, the development of direct electron detectors makes it possible to acquire the entire CBED pattern or Ronchigram during the scanning of electron probe at the speed of $\sim 1000 \mathrm{fps}$ (as shown in Fig. 1A), which could improve the accuracy in the quantification of electron momentum transfer, and provide more details related to the atomic structure. However, from previous experiments and theoretical calculations, quantitative correlation between DPC and the electric field remains a challenge [2], especially when the thickness of the sample is over $4 \mathrm{~nm}$, and STEM samples can routinely be thicker than $20 \mathrm{~nm}$.

We carried out a study on the calculation of electric field based on the measured average momentum transfer of electrons using simulated scanning CBED (convergent beam electron diffraction) patterns in STEM. A $\mathrm{SrTiO}_{3}$ atomic structure is employed as the model system. As demonstrated in previous studies [2], bright field, annular bright field, and high angle annular dark field STEM images can be reconstructed from the scanning CBED patterns. The momentum transfer map can also be calculated using the shift of center of weight (CoW) of each CBED pattern. In this map, oxygen atoms could be revealed regardless of the thickness of the atomic model, as shown in Fig. 1B-C. However, the average momentum transfer is not seen to increase uniformly around all atomic columns as the thickness of the model increases. At 0.4 $\mathrm{nm}$, corresponding to the thickness of a unit cell, the shift in CoW always points outwards from the atomic columns (Fig. 1B). However, at $8 \mathrm{~nm}$, while the shift in CoW near oxygen atoms has only increased in magnitude, the shift close to $\mathrm{Sr}$ and Ti sites has switched direction (Fig. 1C).

To investigate the effect of sample thickness on the shift in CoW and to understand such changes, we have examined the 3D trajectory of the propagating electron probe in the model structure, using a multislice simulation, which allows us to locate the position of the electron probe at each thickness. As shown in Fig. 2 , from our preliminary results, the electron probe does not propagate following a straight pathway; when the initial probe position is near an oxygen column, the probe begins to shift away when it reaches a depth of $4 \mathrm{~nm}$. However, when the initial position is close to $\mathrm{Sr}$ and $\mathrm{Ti}$, the electron probe deviates even earlier. The electron momentum transfer measured using the exiting CBED pattern is therefore the average electric field along the path, not the localized average electric field where the electron probe enters the sample surface [3]. 


\section{References:}

[1] N. Shibata et al, Nature Physics 8 (2012), p. 611.

[2] K. Müller-Caspary et al, Ultramicroscopy (2016).

[3] This work is supported by the NSF with Grant No. DMR 1506535.

A

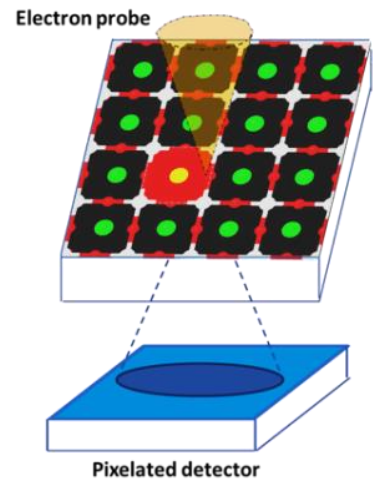

B

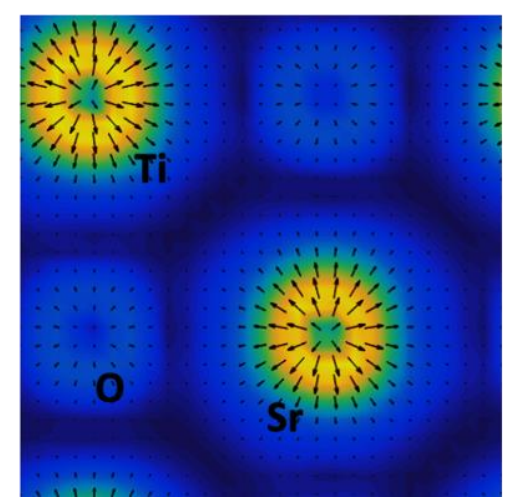

C

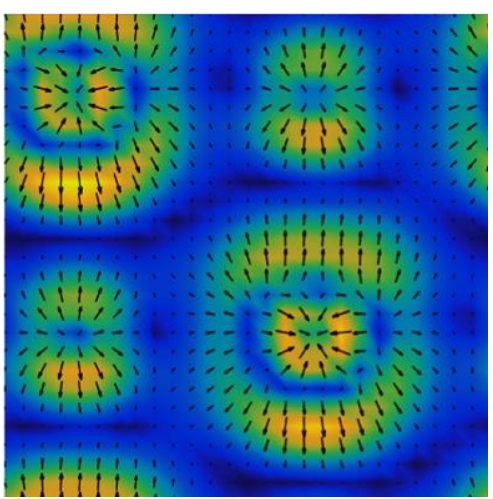

Figure 1. (A) The schematic of using a pixelated electron detector to collect CBED patterns in STEM. (B,C) The reconstructed electron momentum transfer maps from simulated CBED patterns using a $\mathrm{SrTiO}_{3}$ structure model of $0.4 \mathrm{~nm}(\mathrm{~B})$, and $8 \mathrm{~nm}(\mathrm{C})$ thicknesses.
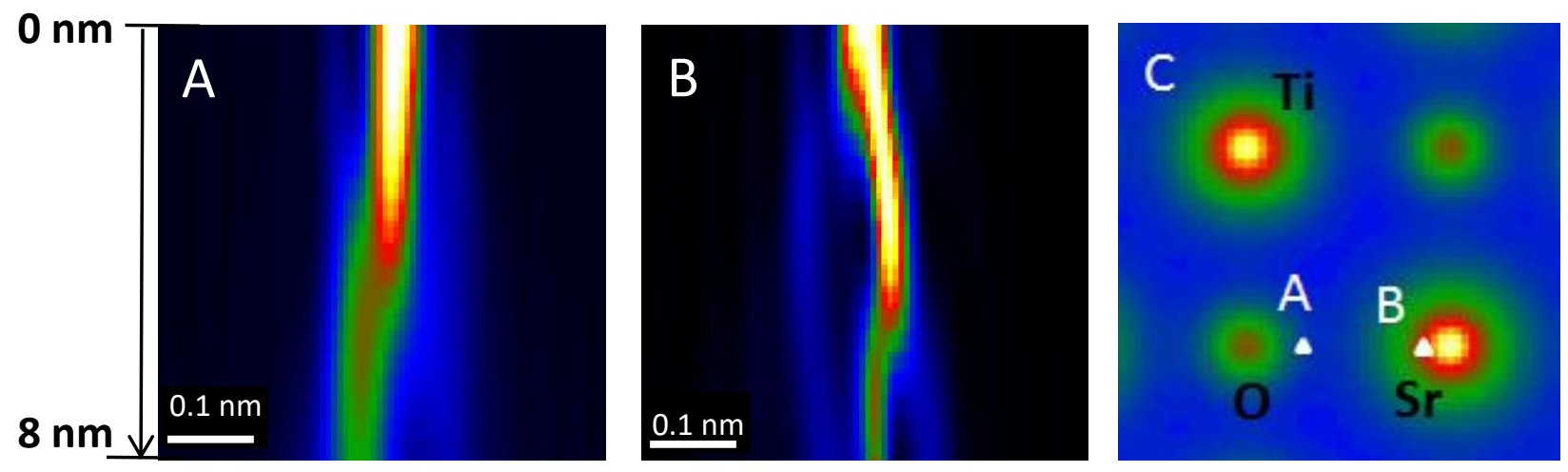

Figure 2. The reconstructed trajectory from multislice simulation, showing the propagation of the electron probe within an $8 \mathrm{~nm}$ thick $\mathrm{SrTiO}_{3}$ sample. The probe profiles in (A) and (B) are from the marked positions in the calculated atomic potential in (C). 